\title{
Cantilever Experiments Update Description of Thermal Noise
}

\author{
Multiple sources of mechanical dissipation seem to explain why a \\ cantilever subject to an extreme temperature gradient has less thermal \\ noise than theory predicts.
}

\section{By Erika K. Carlson}

A ssuming that a system is at thermal equilibrium is a popular physics approximation. But in the real world, that condition is rarely met. Now, Alex Fontana of the École Normale Supérieure in Lyon, France, and colleagues have updated the fluctuation-dissipation theorem (FDT)-a tool for estimating the thermal noise of a physical system-so that it can correctly describe the thermal noise of an out-of-equilibrium microcantilever [1].

In past experiments, researchers had seen that a silicon microcantilever that was significantly hotter at one end than the other vibrated as if it was a room-temperature object in thermal equilibrium. The temperature gradient in these experiments was several hundreds of degrees across a few hundred micrometers. Applying FDT to this out-of-equilibrium system, researchers predict mechanical fluctuations much higher than those the experiments found. In new experiments, Fontana and colleagues pushed a cantilever to a further

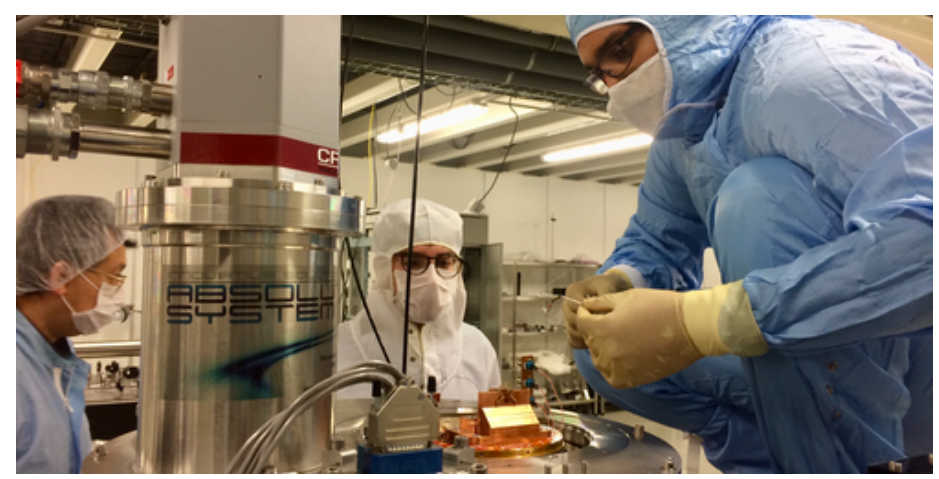

Credit: L. Bellon/ENS de Lyon extreme. They cryogenically cooled the base of a millimeter-long cantilever and heated its tip to its melting point, giving the cantilever ends a temperature difference of about $1700 \mathrm{~K}$. With this experiment, the researchers showed a dearth of fluctuations somewhat similar to the past work.

Fontana and colleagues propose a theoretical framework to explain the phenomenon, which involves updating the FDT. They show that they can fix the theory-experiment fluctuation discrepancy by including a mechanical dissipation with two components. The first component is a "clamping" loss-a loss of mechanical energy through the cantilever's base. The second is "distributed damping," which is a loss of mechanical fluctuations all along the length of the cantilever.

The researchers say that their results could help improve precision measurements affected by mechanical fluctuations, such as the interferometers used in gravitational-wave detectors.

Erika K. Carlson is a Corresponding Editor for Physics based in New York City.

\section{REFERENCES}

1. A. Fontana et al., "Thermal noise of a cryocooled silicon cantilever locally heated up to its melting point," Phys. Rev. E 103, 062125 (2021). 